\title{
Germanica
}

\section{Nelly Sachs ou le refuge de la langue}

Nelly Sachs / Sprache als Zuflucht

\section{Georges Ueberschlag}

\section{(2) OpenEdition}

\section{Journals}

Édition électronique

URL : http://journals.openedition.org/germanica/2808

DOI : 10.4000/germanica.2808

ISSN : 2107-0784

\section{Éditeur}

Université de Lille

\section{Édition imprimée}

Date de publication : 30 juin 1987

Pagination : 91-106

ISSN : 0984-2632

\section{Référence électronique}

Georges Ueberschlag, " Nelly Sachs ou le refuge de la langue », Germanica [En ligne], 1 | 1987, mis en ligne le 28 septembre 2018, consulté le 06 octobre 2020. URL : http://journals.openedition.org/ germanica/2808; DOI : https://doi.org/10.4000/germanica.2808

Ce document a été généré automatiquement le 6 octobre 2020.

(c) Tous droits réservés 


\title{
Nelly Sachs ou le refuge de la langue
}

\author{
Nelly Sachs / Sprache als Zuflucht
}

\section{Georges Ueberschlag}

\author{
IHR WORTE, auf, mir nach! \\ und sind wir auch schon weiter, \\ zu weit gegangen, geht's noch einmal \\ weiter. zu keinem Ende geht's. \\ Ingeborg Bachmann, \\ Für Nelly Sachs, die Freundin
}

116 mai 1940. Le bimoteur en provenance de Berlin vient d'atterrir, enfin, sur l'aéroport de Bromma à Stockholm. Il se fait déjà tard, mais le ciel scandinave, à l'approche de la Saint-Jean, est encore d'une luminosité et d'une transparence extraordinaires. La douceur du soir contraste avec les deux figures chétives qui viennent de descendre de l'avion, deux femmes, deux réfugiées juives, Nelly Sachs et sa mère, qui ont pu échapper à la dernière extrémité à l'enfer de la persécution.

2 Nelly Sachs, alors âgée de 49 ans - elle est née à Berlin en 1891 -, avait reçu quelques jours auparavant l'ordre de se présenter aux autorités allemandes pour être envoyée dans un «camp de travail ». Mais son amie Gudrun Harlan, qui avait des amitiés littéraires et des contacts en Suède, avait encore pu obtenir pour elle - par quel miracle et minuit déjà passé ! - l'autorisation de quitter le territoire allemand. Elle s'était adressée pour cela à Selma Lagerlöf et au prince Eugène Bernadotte, un mécène des lettres, les priant d'intervenir. Selma Lagerlöf, déjà vieille et malade, mourra ce même mois de mai 1940, de sorte que c'est essentiellement à l'intervention du prince Bernadotte que Nelly Sachs doit cette chance d'avoir pu émigrer à la dernière minute.

Elle voulait partir en Amérique, mais les moyens matériels lui manquaient. À Stockholm la communauté juive de la ville lui trouva donc un petit logement, Bergsundsstrand 23, face au lac Maelar. S'y étant installée avec sa mère, elle l'occupera pendant trente ans, jusqu'à sa mort en 1970.

4 Nelly Sachs était arrivée sans bagages. Le seul trésor qu'elle avait pu sauver, son seul bagage, c'était sa langue, la langue allemande, celle de ses bourreaux, mais qui allait 
aussi devenir celle de son génie. Elle y trouva un refuge, une compensation pour sa patrie perdue, un domicile hors d'atteinte.

5 C'est ici, à Stockholm, en exil, qu'elle connut sa véritable naissance à la poésie, une naissance au milieu des larmes et de la mort. «Der Tod war mein Lehrmeister», écritelle dans Leben unter Bedrohung, un des rares fragments autobiographiques qu'elle nous a laissés.

6 Certes, Nelly Sachs avait déjà fait auparavant ses débuts littéraires à Berlin. Fille unique du riche industriel William Sachs, elle avait connu une enfance heureuse, choyée par les siens dans leur villa de Grünewald. Son père, qui n'était guère attaché à la tradition religieuse juive, croyait, en revanche, à l'idéal humaniste de Goethe et au progrès technique. Sa petite fille l'accompagnait très tôt lorsqu'il jouait du piano, «à la recherche de ces contrées où la langue n'a plus cours». L'enthousiasme pour la musique et la poésie de la jeune Nelly n'avait d'égal que son amour de la danse, dans laquelle elle trouva ses premières possibilités d'expression.

7 Pour son quinzième anniversaire ses parents lui offrent La Légende de Gösta Berling de Selma Lagerlöf. Elle se plonge avec ravissement dans l'œuvre de la grande Suédoise et sent naître le désir de rivaliser avec elle. En 1921 elle lui envoie son premier ouvrage imprimé, Legenden und Erzählungen, avec une dédicace où elle exprime l'admiration d'une jeune Allemande qui voit dans la grande poétesse suédoise son lumineux exemple. Selma Lagerlöf lui fit parvenir un mot de remerciement, et Nelly Sachs continua à rester en relations avec elle, lui envoyant notamment les poèmes qu'elle avait commencé à écrire depuis l'âge de dix sept ans ${ }^{1}$.

8 Ces poèmes, écrits par une jeune fille de la bonne bourgeoisie qui a parfaitement accepté et assimilé les règles de vie et l'idéal de son milieu conservateur, ne cadrent guère avec l'actualité littéraire berlinoise d'alors, bien qu'ils se détachent nettement du lyrisme féminin que l'on rencontre habituellement dans des cercles qui se piquent de littérature. Les nombreux sonnets qu'on y trouve témoignent déjà d'une volonté de la forme, d'une expression et d'une maitrise de la langue tout à fait classiques. Quant à leurs thèmes, ils sont proches du romantisme, Nelly Sachs se situant volontiers dans la mouvance poétique de Novalis et vouant à la langue de Hölderlin une profonde admiration.

9 Mais ces poèmes semblent totalement ignorer encore l'aventure moderne de la langue, de l'expression poétique. Dans un Berlin qui était devenu un des centres du mouvement expressionniste, Nelly Sachs continue à vivre dans son propre univers poétique, à l'écart, loin des milieux bohèmes où se complaisent ses compatriotes.

Les titres de ces cycles de poèmes, dont la Vossische Zeitung sera la première à publier des extraits - d'autres paraîtront dans les revues Jugend et Der Morgen ${ }^{2}$, sont déjà très significatifs: Leise Melodie, Melodien der Bibel, Lieder vom Abschied. Les images et les thèmes sont imprégnés de romantisme: la nature, la musique, Dieu, l'amour et la mort. La musique surtout, celle de Mozart et de Boccherini, égrène de poème en poème son message de la parenté universelle et de la nostalgie des sphères sublimes: «Heimwehdunkel sehnt Klang um Klang sich fort» ${ }^{3}$. 
11 Dans sa ferveur religieuse et panthéiste Nelly Sachs devine le processus de la transformation, de la transcendance, elle le décrit de l'extérieur, mais elle ne le vit qu'au stade du désir. Elle manque manifestement encore de souffle, mais de précieux encouragements lui parviennent déjà. Stefan Zweig, qui la remercie pour quelques poèmes reçus en 1925, souligne la remarquable "progression extatique » qu'il y discerne. Plus tard la métamorphose - die Verwandlung, un des mots clés de sa poésie s'opèrera concrètement dans l'écriture.

Dans ses premières tentatives poétiques ${ }^{4}$, Nelly Sachs, loin des tendances expressionnistes ou surréalistes de son époque, ne soupçonne encore ni les possibilités ni les problèmes d'une rénovation radicale de la langue. Le vocabulaire poétique, avec ses «Königswörter» n'a rien de problématique pour elle. Ses poèmes, conformes aux canons du romantisme allemand, de facture très conventionnelle, semblent donc refléter jusque vers 1934 un univers à l'abri des bouleversements, extérieurs et intérieurs.

13 Mais déjà ce calme est trompeur. La mort de son père en 1930 a arraché Nelly Sachs assez brutalement à sa situation de confort et de sécurité matériels et la montée du péril nazi la rejette dans un isolement culturel qui, littéralement, la paralyse et la dépayse - officiellement elle n'est plus considérée comme allemande, mais comme juive - et qui la plonge dans des sentiments inconnus jusqu'alors, la peur, l'horreur et l'angoisse. Le seul refuge qui s'offre à elle dans ces instants, ce sont les livres de sagesse dont la bibliothèque paternelle était largement pourvue, ceux de l'Ancien Testament, les livres de la Cabale et particulièrement le Livre de Sohar.

14 Jusqu'à ce jour de mai 1940 où, la mort s'apprêtant déjà à la saisir, elle échappe de justesse à l'anéantissement complet.

15 En Suède, sur cette terre d'asile et de triste espérance, Nelly Sachs connaît, sans l'avoir vraiment voulu, une nouvelle naissance poétique. Après une longue période de maturation et lorsqu'elle en avait pratiquement déjà abandonné l'espoir, elle renaît à la poésie, de manière explosive presque.

La force qui rend possible cette renaissance, et qui l'incarne entièrement, c'est la langue précisément, le seul bien qui lui reste après sa fuite et qui n'est elle-même qu'une patrie en lambeaux, où les demeures ne sont plus que décombres fumants.

17 Cette langue, la langue allemande, non seulement elle ne l'a pas reniée en partant ${ }^{5}$, non seulement elle n'en a pas fait la langue de la vengeance, mais elle la sauve des bourreaux en en faisant la langue des victimes, de ceux qui séjournent "dans les demeures de la Mort $»^{6}$. La fuite en Suède s'accompagne d'une fuite au plus profond de l'essence même de la langue, là où les mots eux-mêmes s'évanouissent, là où il ne reste que le mystère.

18 Car la langue poétique de Nelly Sachs ne relèvera d'aucune technique à proprement parler, d'aucun Verfremdungseffekt, elle ne sera ni code ni chiffre, elle sera ce reliquat, cette blessure parfois étrange et baroque que laisse toute énigme derrière elle lorsqu'on a tenté de la pénétrer, surtout l'énigme du Mal et de la souffrance. 
Ce n'est qu'au cours de l'hiver 1943, à une époque où elle s'est déjà assise à l'ombre de la Mort, où elle scrute intensément les contours de l'éternité, de ce qu'elle appellera dans ses poèmes «das Staublose», qu'elle recommence à écrire. Entre temps elle s'était accoutumée à la langue suédoise, l'apprivoisant comme on apprivoise le chant du rossignol, s'accrochant à quelques bribes de cette terre d'asile et se nourrissant, comme elle le dit elle-même, de cette langue apprise dans les larmes :

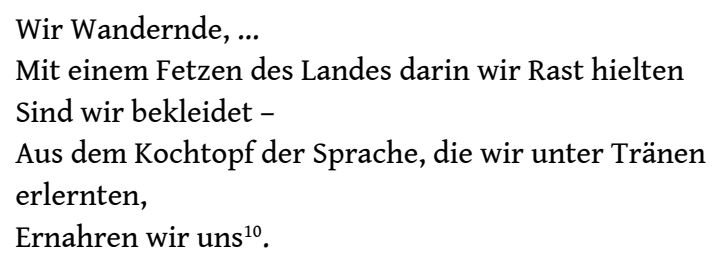

Mais cet apprentissage lui ouvre des horizons nouveaux et déterminants. Elle lit les poètes suédois contemporains, Karl Vennberg, Erik Lindegren, Dan Andersson, Johannes Edfeld, Edith Södergran, Ragnar Thoursie, se lie d'amitié avec quelques-uns et se met à traduire leurs poèmes en allemand ${ }^{11}$. La traduction devient pour elle un acte de re-création, un « acte critique », selon l'expression de Paul Valéry.

Et sous cette double influence, sous cette double commotion que représentaient l'angoisse de la mort et celle de l'exil, et la révélation, grâce à la poésie moderne suédoise, d'un lyrisme aux possibilités d'expression insoupçonnées ${ }^{12}$, son génie latent secoue ses chaînes et elle se met à écrire. Fébrilement. Pour survivre.

L'exil donne à sa langue maternelle une résonnance et une profondeur nouvelles. La distance qui s'est temporairement établie l'incite à une appréhension neuve et 
originale du vocabulaire. Une langue de feu et de sang jaillit sous sa plume - «Ich schrieb wie in Flammen $»^{13}-$, révélation prophétique d'une force cosmique, une langue épousant presque inconsciemment celle de la Cabale, de la Thora et des légendes des Chassidims.

Da schrieb der Schreiber des Sohar

und öffnete der Worte Adernetz

und führte Blut von den Gestirnen ein ${ }^{14}$,

c'est ainsi que commence un de ses cycles de poèmes. Il est évident que Nelly Sachs voit dans l'auteur du livre de Sohar l'archétype du poète, celui qui a percé le mystère de l'alphabet, de la création et du devenir du monde.

L'alphabet, l'écriture, le mot élémentaire, autant de notions qui reviennent toujours dans la poésie de Nelly Sachs, ne désignant pas tant les matériaux dont est faite toute littérature que des stigmates de la Nature, qui entraînent le moindre détail, l'insignifiant, le sable et le papillon, dans une relation cosmique.

Ce qui frappe le lecteur de Nelly Sachs, et les rares critiques qui se sont penchés sur son œuvre, c'est l'extraordinaire ampleur de sa langue poétique, une langue qui embrasse dans un même souffle les espaces et les époques, qui s'élève sans peine du plus petit à l'infiniment grand. Pour celle qui se considère comme appartenant à la grande cohorte des persécutés - «die Gejagten der Zeit»-, c'est le refuge dans lequel sa pauvre existence terrestre $\mathrm{e}^{15}$ prend un autre sens et arrive à se transcender.

Les métamorphoses du monde lui tiennent lieu de nouvelle patrie - «An Stelle von Heimat halte ich die Verwandlungen der Welt» dit-elle dans l'épitaphe qui introduit son recueil de poèmes Flucht und Verwandlung. Bien plus, la langue elle-même, dépassant la sphère du particulier pour atteindre l'universel, devient métamorphose du monde, le refuge hors de l'espace et du temps dans lequel elle arrive à dompter les horreurs d'un monde sorti de ses gonds ${ }^{16}$.

31 Malgré la célèbre parole de Théodore Adorno, disant qu'après Auschwitz il n'était plus possible de faire de la poésie, Nelly Sachs et sa langue témoignent de la permanence poétique, de la permanence du poïein, de la force créatrice. Mort, où est ta victoire ? tel est le défi qu'elle lance au pessimisme de ses contemporains.

Certes, durant les quinze années de sa brève et intense production poétique, la langue de Nelly Sachs a évolué. Mais ce n'est jamais une langue que l'on pourrait qualifier d'harmonieuse. L'auteur elle-même l'appelle son cri muet ${ }^{17}$. Faut-il en chercher la racine dans l'expressionnisme allemand, dans le caractère extatique et visionnaire de cette langue, comme Horst Bieneck le fait un peu rapidement ${ }^{18}$ ? Cela est très peu vraisemblable, et pas uniquement en raison de la distance volontaire dans laquelle Nelly Sachs s'était tenue vis-à-vis de ce mouvement, voire de l'indifférence qu'elle avait manifestée à son égard.

33 Car, même si elle est un des rares poètes de l'après-guerre, avec Gottfried Benn, à avoir assimilé, et même renouvelé, les canons de la langue de l'expressionnisme, elle va bien au delà, ne se laissant ranger dans aucun -isme. Se nourrissant de la tradition biblique juive et de l'expression formelle du lyrisme suédois moderne, comme nous l'avons déjà 
souligné, elle se montre également très avertie de la manière dont le surréalisme use des images.

À aucun moment la langue ne devient pour Nelly Sachs un objet d'expérimentation. Elle est son vécu immanent. À mesure que le processus de la création poétique se développe, son expression devient plus concise, plus radicale, se rapprochant de ce que l'on considère comme la grande caractéristique du lyrisme moderne, le «cri silencieux ", la tendance à s'incliner et à se taire devant l'ineffable. L'évolution que l'on constate entre les poèmes du début et les derniers vers de Glühende Rätsel ${ }^{19}$ est, à ce propos, éloquente. La forme, et surtout la préoccupation de perfection formelle, sont progressivement reléguées à l'arrière-plan, voire effacées devant l'intensité de l'événement vécu. Les derniers poèmes de Hölderlin avaient déjà montré cette parfaite réduction à l'essentiel. C'est à lui, à cet exemple lumineux pour Nelly Sachs, que remonte d'ailleurs la lignée des poètes modernes, Trakl vers la fin de sa vie, Rilke, Paul Celan, qui ne se réfèrent à aucun programme poétologique et pour qui l'éclatement de la syntaxe et du vocabulaire n'a qu'un seul but, reculer les limites de l'indicible pour se rapprocher de l'essentiel, du divin.

La voix qui se révélait à Moïse dans le buisson ardent - Ego sum qui sum - est, à cet égard, l'idéal inégalable de cette langue qui déjà bascule et qui pourtant se meut encore sur la crête effilée de ses propres limites, de cette langue qui n'existe que par ses extrêmes, selon la formule de Paul Celan - «Das Gedicht behauptet sich am Rande seiner selbst $»^{20}$.

Cette conception d'une langue poétique transmuée a, chez Nelly Sachs, des sources mystiques. Elle se rattache à la tradition gnostique à laquelle l'auteur se réfère très fortement. C'est une tradition qui va, pour elle, de Jérémie à Jakob Böhme, passant par le Christ, le Livre de Sohar et les Chassidims. La notion gnostique de la transmutation se conçoit par rapport à celle de l'«Ungrund», elle exclut l'idée de constance, d'achevé, de demeure ou de patrie. Il est donc tout à fait évident que la langue ne peut se concevoir pour Nelly Sachs qu'en devenir. Le célèbre «Stirb und werde» de Goethe est repris par elle dans une formule tout aussi lapidaire : «hindurchsterben $»^{21}$. Elle rêve donc à la fin de sa vie, dans les Glühende Rätsel en particulier, d'une langue sans mots, d'une langue qui ne serait plus que souffle ${ }^{22}$.

Le sens de ce devenir, de cette évolution de la langue est magnifiquement illustré par les titres mêmes, très significatifs, des recueils poétiques de Nelly Sachs. Depuis In den Wohnungen des Todes (1947), notion baroque qui rappelle que dans la tradition juive le cimetière est aussi appelé "la maison de la vie", s'élève une ligne abrupte: Sternverdunkelung (1949), Und niemand weiss weiter (1957), Flucht und Verwandlung (1959), jusqu'à ce recueil Fahrt ins Staublose (1961) qui donne son titre et son sens à l'édition complète des œuvres de Nelly Sachs ${ }^{23}$.

Et dans le dernier cycle de poèmes, Die Suchende (1967), écrit après les Glühende Rätsel à l'approche de ses 80 ans, nous trouvons encore un dernier écho au Hindurchsterben, venu d'un ailleurs déjà tout proche. Les visions et les larmes, y lisons-nous, écriture zigzagante de la foudre, ces pèlerins venus de l'ailleurs, ne commencent à nous parler qu'à la naissance de la mort :

... aber Tränen und Visionen

Wahnsinn und Schrift der Blitze

diese Flüchtlinge von anderswoher 
warten bis Sterben ist geboren

dann reden sie -24

39 La progression poétique vers les extrêmes de la langue ${ }^{25}$, vers la transmutation, affecte tout naturellement la forme même des poèmes. Ces textes en rythmes libres, qui ne se plient qu'en apparence, ou pas du tout, aux contraintes de la strophe - les rimes ont disparu depuis longtemps -, qui évoquent d'abord les hymnes de Hölderlin, s'achèvent dans des formules lapidaires, dans le staccato et les répétitions anaphoriques. Toute liaison, toute motivation, tout enchaînement, logique ou non, à fortiori tout décor et toute rhétorique en sont absents.

Le poème devient ainsi non pas chiffre, mais à proprement parler une réduction, et risque de déboucher sur l'impuissance, voire l'échec poétique. La magie de la langue recule les horizons, certes, mais elle les recule seulement.

41 Les procédés stylistiques que Nelly Sachs emploie, les compositions nominales, les hyperboles, les figures antithétiques, les interférences du concret et de l'abstrait, les possibilités multiples des formes verbales, les synecdoques et surtout les métaphores, tout cela n'est pas nouveau dans la poésie moderne. Souvent même ces procédés y sont galvaudés. Mais Nelly Sachs leur donne une pureté, elle les emploie avec une retenue et une justesse qui les élève. Les poncifs et les clichés sont à cent lieues, les mots perdent leur proximité matérielle, les images leur sens, le signifiant s'évade et échappe au signifié qui nous reste entre les doigts. Le concret n'est plus qu'un tremplin qui permet à la langue de se sublimer, de s'élever dans les sphères où son enveloppe matérielle, la «Worthülse» dit Nelly Sachs, s'évanouit, où s'opère la Entdinglichung.

Si Nelly Sachs parle de Rätselrinde, Spirale der Eile, si elle emploie des expressions comme nachtbehandschuht, Treibeis von Tod und Auferstehung, etc. - et la liste en serait bien longue, impossible même à établir sans englober la quasi totalité des poèmes et sans se référer au contexte sans lequel tout exemple cité ne peut que prendre les apparences d'une pièce de collection -, elle ne cède pas à cette mode facile, répandue dans les années 1950, qui consistait en poésie à établir des connexions arbitraires et à mettre tout en rapport avec tout ${ }^{26}$.

Pour qualifier cet aspect de la langue de Nelly Sachs, qui n'a rien à voir avec toutes les métaphores faites au pochoir, Hellmut Geissner a sans doute trouvé l'une des expressions les plus justes lorsqu'il parle de «evozierende Metaphorik» ${ }^{27}$.

Le vocabulaire fondamental dont se sert Nelly Sachs, du fait même du caractère ascendant de sa langue - «eine extatisch aufsteigende Linie» disait déjà Stefan Zweig en 1925 - est d'une extrême élémentarité. Ce sont pour la plupart des substantifs monosyllabiques - Sand, Mensch, Tod, Fisch, Tanz, Licht, Wind, Stern, etc. - que l'auteur emploie dans leur complémentarité antithétique, afin de conjurer la confusion, la perturbation, la déroute linguistique qui menace le genre humain: "O dass nicht einer Tod meine wenn er Leben sagt» ${ }^{28}$. L'entreprise poétique de Nelly Sachs consiste donc aussi à sauver, littéralement, la langue, une langue que les hommes auraient perdue. Une légende juive raconte que lors du Déluge la langue des hommes elle-même aurait été engloutie dans les flots, de sorte qu'il avait fallu recréer, à la sortie de l'Arche, un nouvel alphabet, redonner aux mots leur sens premier qu'ils avaient perdu 
dans un monde dévoyé. On voit très bien la comparaison qui a pu s'imposer à Nelly Sachs avec sa propre époque, et elle a fait de cette légende le thème d'un de ses poèmes scéniques, Beryll sieht in der Nacht, dont le sous-titre, ô combien ramassé et significatif, résume sa propre mission poétique : «Das verlorene und wieder gerettete Alphabet».

Nelly Sachs ne cherche cependant pas à innover, surtout pas. Elle n'opère aucune critique directe et on ne l'imagine pas écrivant un essai sur l'état ou la dépravation de la langue. Elle veut sauver ce qui peut l'être et elle ramasse religieusement les "Worthülsen», les dépouilles de la langue sur lesquelles elle est assise dans son exil comme jadis Job sur son tas de cendres, pour y découvrir la chrysalide dont naîtra le nouvel alphabet, le nouveau " cosmos des mots». Elle adjure donc les peuples de la terre, qui se délectent du miel de la confusion verbale, de ne pas détruire ce cosmos :

Völker der Erde...

die ihr in die Sprachverwirrung steigt

wie in Bienenkörbe,

um im Süssen zu stechen

und gestochen zu werden -

Völker der Erde,

zerstöret nicht das Weltall der Worte ${ }^{29}$.

Et dans ce même poème elle les exhorte, ces peuples de la terre, à ne pas trahir, à ne pas chasser les mots de leur source mystique, car le mot participe activement à la cosmogonie, il « aide à engendrer les astres ».

S'appuyant ainsi, comme nous l'avons déjà souligné, sur la théorie gnostique de la langue, dont la chaîne d'influences remonte aux Temps anciens, et dont il serait aléatoire de vouloir disséquer ou analyser l'importance des maillons pour elle, Nelly Sachs conçoit la parole, le parler, comme une création. Au début était la Verbe. Elle en condense l'idée dans l'image du «Zungenbaum». L'Arbre du savoir, dressé jadis dans le Paradis, est aussi l'Arbre de la langue.

Les poèmes de Nelly Sachs ont donc une double dimension, ils nécessitent une lecture à des niveaux différents qui s'interpénètrent constamment. Une échelle s'établit, allant $\mathrm{du}$ sens historique, qui, en retraçant l'holocauste du peuple juif, compose une page $\mathrm{du}$ grand livre de la souffrance humaine, au sens mystique grâce auquel la langue entre dans le processus de la création.

Et l'alphabet, tel un ange ressuscité, le «Buchstabenengel» comme il est dit dans le poème «Sohar: Schöpfungskapitel», déroule son linceul en libérant les signes de la Nature :

Des Alphabetes Leiche hob sich aus dem Grab,

Buchstabenengel, uralter Kristall,

mit Wassertropfen von der Schöpfung eingeschlossen...

Und wickelt aus, als wärens Linnentücher, darin Geburt und Tad ist eingehüllt, Buchstabenleib, die Falterpuppe aus grüner, roter, weisser Finsternis. 
L'essence de la langue ne peut être, c'est clair, que d'ordre religieux.

La langue ouvre ainsi à Nelly Sachs la porte d'un refuge, d'une nouvelle patrie qui ne demande pas de retour. Parce que sa vie a ainsi trouvé une autre dimension, Nelly Sachs s'est accommodée, sans jamais s'en plaindre à notre connaissance, de sa retraite exiguë de Bergsundsstrand 23 à Stockholm. L'exil, ce n'était plus la terre suédoise, mais la terre tout court, et l'impossible retour dans la patrie allemande est devenu l'inutile retour.

Néanmoins, Nelly Sachs retourna à quelques rares reprises, après la fin de la guerre, en Allemagne, en particulier le 17 octobre 1965 pour recevoir à Francfort le Friedenspreis des deutschen Buchhandels. Elle s'y est rendue pour affirmer qu'elle se considérait non pas comme une poétesse juive, mais avant tout comme une Allemande ${ }^{30}$. Faut-il rappeler que c'est en tant que telle qu'elle reçut en 1966 le Prix Nobel de littérature : « Nelly Sachs, poète allemande domiciliée en Suède depuis 1940 », comme il est dit dans le communiqué de l'Académie suédoise, rédigé traditionnellement en français.

3 Certes, il fallait du courage à Nelly Sachs pour s'affirmer jusqu'au bout poète allemande, comme il en avait fallu à Elisabeth Langgässer et à Gertrud Kolmar, alors qu'on avait voulu les chasser de la langue allemande, leur imposant l'interdiction d'écrire, la croix de l'exil ou la souffrance du camp d'extermination. Mais la langue a été leur refuge, elle a rendu possible l'impossible réconciliation, elle a été le vase inaltérable de leur pensée et le signe d'une puissance qui dépasse les artifices du Mal. Comme elle le fut quelques années plus tôt déjà pour leur sœur scandinave Edith Södergran, en quête du " Pays qui n'est pas ».

Il est significatif qu'à la mi-temps du XX $\mathrm{X}^{\mathrm{e}}$ siècle ce sont précisément elles, ces femmes entrânées dans les tourbillons de l'épouvante, qui, en compagnie d'Else Lasker-Schüler et continuant la grande tradition qui passe par Ricarda Huch et remonte à Annette von Droste-Hülshoff, ont donné au lyrisme allemand de nouveaux titres de noblesse. À elles sans doute s'adressent ces vers que Nelly Sachs a tracés, avec son humilité coutumière, dans les Glühende Rätsel :

Wir winden hier einen Kranz

Manche haben Donnerveilchen

ich nur einen Grashalm

voll der schweigenden Sprache.

\section{NOTES}

1. Certains de ces poèmes nous ont été conservés uniquement parce qu'ils figuraient dans les archives que Selma Lagerlöf a laissé à Märbacka.

2. Revue mensuelle publiée à Berlin par le Jüdische Kulturbund.

3. Sachs, N. Frühe Gedichte. Manuscrit de la Nelly Sachs-Sammlung, Archiv Dortmund.

4. Il s'agit d'une véritable éruption du génie poétique telle que l'histoire de la littérature allemande n'en offre que peu d'exemples. Fontane, certes, et quelques autres. 
5. D'autres réfugiés allemands en Suède renonceront définitivement à la langue allemande, espérant ainsi conjurer les cauchemars. Erwin Leiser, l'ami de B. Brecht, Hilde Rubinstein ou la fille d'Elisabeth Langgässer n'écriront plus qu'en langue suédoise.

6. Titre du premier recueil de poèmes de $\mathrm{N}$. Sachs publié après la guerre.

7. Sachs N., Anmerkungen zu Beryll sieht in der Nacht, manuscrit de la Nelly Sachs-Sammlung, Archiv Dortmund.

8. Le Livre de Sohar, qui constitue un commentaire du Premier livre de Moïse et qui est un des écrits fondamentaux de la pensée mystique, de celle de Jakob Böhme en particulier, dit notamment que dans les premiers mots de la Bible, Bereshit bara elohim - Au début créa Dieu, le véritable sujet n'est pas Dieu, mais Bereshit «Au début ", alors que dieu devient objet dans la phrase. Elohim est donc la lumière née de la Lumière, non pas la Lumière primitive elle-même.

9. Sachs N., Ausgewählte Gedichte, Suhrkamp 1963, p. 35.

10. Sachs N., In den Wohnungen des Todes, Chor der Wandernden, Suhrkamp 1965, p. 96.

11. À propos de l'influence du lyrisme suédois sur l'activité créatrice, la langue et la poésie de Nelly Sachs, nous renvoyons à notre article Das Wahlverhältnis der Nelly Sachs zur schwedischen Lyrik, Wissenschaftliche Zeitschrift der Universität Halle, xxxv' 86 G. Heft 1, p. 76-88.

12. «Ohne den Rückhalt dieser schwedischen Dichtung würde sie, glaube ich, nie gewagt haben, den eigenen Eingebungen zu vertrauen». Olof Lager-Crantz, «Mit meinem Untergang nahre ich dich», in Das Buch der Nelly Sachs, Frankfurt/Main 1977, p. 400.

13. Cf. Lagerkrantz O., Versuch über die Lyrik der Nelly Sachs, Frankfurt Main 1967, p. 43.

14. Sachs N., Und niemand weiss weiter, «Sohar Schöpfungskapitel», Hamburg / München 1957.

15. Nelly Sachs a toujours vécu pauvrement dans les quelques $\mathrm{m}^{2}$ de son premier refuge stockholmois, ne fréquentant que quelques rares amis et un petit cercle de poètes. Même l'attribution du Prix Nobel ne changea pas grand chose à sa vie.

16. Bieneck H., «Sprache als Verwandlung der Welt », Nelly Sachs zu Ehren, Suhrkamp, Frankfurt / Main 1966, p. 80.

17. «Das Dichten war mein stummer Schrei », in «Leben unter Bedrohung», Ariel, 1956.

18. Bieneck H., op. cit., p. 83.

19. Quatre cycles de poèmes, in Späte Gedichte, Suhrkamp 1967.

20. Celan Paul, Büchner-Preis Rede, 1960.

21. Sachs N., Une niemand weiss weiter, poème $n^{\circ} \mathrm{l}$, Hamburg, 1957.

22. Cf. Olof Lagerkrantz, Versuch über die Lyrik der Nelly Sachs, Suhrkamp 1967, p. 79.

23. Il est évident que dans le cadre de cet article nous ne pouvons guère nous arrêter au contenu même, à la substance de ces poèmes. Mais le lecteur pourra consulter utilement à ce sujet : Das Buch der Nelly Sachs, 1968 (Beda Alleman, «Hinweis auf einen Gedichtraum», pp. 291-308. Gisela Dischner, «Zu den Gedichten von Nelly Sachs», pp. 309-354). Olof Lagerkrantz, Versuch über die Lyrik der Nelly Sachs, Stockholm 1967. Walter Berendsohn, Nelly Sachs. Einführung in das Werk der Dichterin jüdischen Schicksals, Darmstadt 1974. Peter Sager, Nelly Sachs. Untersuchungen zu stil und Motivik ihrer Lyrik, Diss. Bonn 1970.

24. Sachs N., Die Suchende, Suhrkamp 1967.

25. Il faudrait aussi y inclure les pièces dramatiques que Nelly Sachs a composées, surtout Eli. Ein Mysterienspiel vom Leiden Israels (1951) et Verzauberung (1970).

26. Il s'agit là sans doute d'un aspect de ce phénomène souvent vérifié au cours de l'histoire, à savoir la véritable explosion baroque qui suit toute grande catastrophe.

27. Geissner H., « Nelly Sachs », Deutsche Literatur seit 1945, Hrsg. von Dietrich Weber, Stuttgart 1971, p. 28.

28. Sachs N., Ausgewählte Gedichte, Suhrkamp 1963, p. 29.

29. Ibid. 
30. Cf. à ce sujet Olof Lagercrantz qui écrit en 1967 dans son essai Versuch über die Lyrik der Nelly Sachs: «Es ist Zeit, dass man in Deutschland begreift, dass sie eine Landsmännin ist, die vertrieben wurde, und keine Fremde, die man zur Ehrenbürgerin macht».

\section{RÉSUMÉS}

Échappant à la dernière extrémité à la persécution nazie, Nelly Sachs se réfugie à Stockholm en mai 1940, y amenant comme seul bagage sa langue maternelle. Celle-ci deviendra pour elle comme un domicile hors d'atteinte, compensation pour une patrie perdue.

C'est à Stockholm qu'elle connaît sa véritable naissance poétique, sous le double choc de sa rencontre avec la mort et avec la poésie moderne suédoise, dont elle traduit des extraits en allemand. La familiarité avec les mystiques, acquise durant les années d'oppression, la conforte dans son élan vers le mystère originel de la langue. L'exil donne à celle-ci une résonnance nouvelle et l'incite à une appréhension neuve du vocabulaire. Le mystique devient l'archétype du poète, celui pour qui l'alphabet - «das verlorene und wieder gerettete Alphabet» - ne représente plus que des stigmates de la Nature, entraînant le moindre détail dans une relation cosmique.

Ampleur de la langue poétique de Nelly Sachs, que l'auteur appelle son cri muet et dont il ne faut chercher les racines ni dans l'expressionnisme ni dans aucun autre -isme, mais dans le vécu immanent qui relègue le souci de la forme à l'arrière-plan. Poésie qui est parfaite réduction à l'essentiel, au divin.

Même si cette langue poétique transmuée, où s'opèrent les métamorphoses du monde, se sert de procédés stylistiques connus, de la métaphore surtout, elle les élève dans les sphères où l'enveloppe matérielle du mot s'évanouit. Il reste la chrysalide dont naîtra le nouvel alphabet, en libérant les signes de la Nature. La langue, créatrice, est donc d'essence religieuse et ouvre ainsi à l'auteur la porte d'un refuge, d'une patrie qui ne demande pas de retour.

Nach ihrer im Monat Mai 1940 noch gelungenen Flucht nach Stockholm beugt sich Nelly Sachs, als Spätgeborene im Land der Dichtung, über das ihr einzig noch verbliebene Hab und Out, ihre deutsche Muttersprache, und sie findet darin eine neue Zuflucht. Unter dem doppelten Eindruck des «Lehrmeisters Tod» und der modernen schwedischen Lyrik, die sie übersetzt, erlebt sie ihre wirkliche dichterische Geburt.

Der schon 1935 einsetzende Umgang mit den Mystikern hat ihr die «Fahrt ins Staublose» (Titel eines ihrer Gedichtzyklen) ermöglicht, dort wo die Sprache, das «verlorene und wieder gerettete Alphabet», in ihrem schöpferischen Geheimnis und in ihrem kosmischen Zusammenhang erfasst werden kann.

Die weit sich ausspannende poetische Sprache der Nelly Sachs, die am Ende nur noch stummer Schrei der Dichterin bleibt, steht trotz ihrer Zeitgebundenheit kaum in einer historischen Kontinuität. Sie lässt sich weder auf den Expressionismus noch auf irgend einen anderen - Ismus reduzieren, sondern wird und wirkt aus dem immanent Erlebten. Das Bedürfnis der Form und das Suchen nach neuen Ausdrucksmöglichkeiten ersterben hinter dem allein Wesentlichen der Poesie, des «Zungenbaums», hinter dem Mitwirken am göttlichen Schöpfungsprozess.

Stilistisch oft gebrauchte Mittel, manchmal schon verbrauchte, besonders die Metapher, werden bei Nelly Sachs in eine Sphäre erhoben, wo die «Worthülsen» fallen und das neue Alphabet in einer freirythmischen Versform den Kranz des Gesanges um tote Dinge winden kann, sie zu neuem Leben erweckend. Die gnostische, sowie die christlich-mystische Erfahrung der Sprache 
bei Nelly Sachs, öffnet ihr die Tür einer neuen - göttlichen - Heimat, wo die Dichterin keiner Um - noch Rückkehr mehr bedarf. Statt Ersatz wurde ihr das Wesen zuteil.

\section{AUTEUR}

\section{GEORGES UEBERSCHLAG}

Université de Lille III 